\title{
抗癌药物达卡巴嗪的合成工艺优化
}

\author{
肖胜伟 $a$ 刘志军 ${ }^{a}$ 柳杨 ${ }^{a}$ 陈河如*,,$b$ \\ $\left({ }^{a}\right.$ 暨南大学药学院中药及天然药物研究所 广州 510632) \\ $\left({ }^{b}\right.$ 广东省中药药效物质基础及创新药物研究重点实验室 广州 510632)
}

\begin{abstract}
摘要 以廉价易得的甘氨酸为原料，经酯化、甲酰化、脱水、酰氨化得到异氰基乙酰胺，四步总收率 $42.9 \%$. 异氭基乙 酰胺与氨基腈在碱性条件下环合得到重要中间体 5-氨基-4-咪唑甲酰胺，收率 $68.2 \%$. 随后经重氮化，与二甲胺偶联，得 最终产物达卡巴嗪, 总收率 $22.3 \%$. 所有中间体以及目标产物的结构均由 ${ }^{1} \mathrm{H}$ NMR, ${ }^{13} \mathrm{C}$ NMR 和 ESI-MS 确证. 合成路线 具有原料廉价、反应条件温和、总收率较高等特点, 是一条经济实用的合成工艺路线.

关键词＼cjkstart达卡巴嗪；工艺优化；药物合成；抗癌
\end{abstract}

\section{Optimization of the Synthetic Process of Antineoplastic Drug Dacarbazine}

\author{
Xiao, Shengwei $^{a} \quad$ Liu, Zhijun $^{a} \quad$ Liu, Yang $^{a} \quad$ Chen, Heru $^{*, a, b}$ \\ ( ${ }^{a}$ Institute of Traditional Chinese Medicine and Natural Products, College of Pharmacy, \\ Jinan University, Guangzhou 510632) \\ ( ${ }^{b}$ Guangdong Province Key Laboratory of Pharmacodynamic Constituents of TCM and New Drugs Research, \\ Jinan University, Guangzhou 510632)
}

\begin{abstract}
Dacarbazine has been prepared started from glycine, which was carried on firstly through a four-step process including esterification, formylation, dehydration, amidation to give 2-isocyanoacetamide with a total yield of $42.9 \%$. 2Isocyanoacetamide was then cyclized with cyanamide under basic condition to afford the important intermediate 5-amino- $1 H$-imidazole-4-carboxamide with yield of $68.2 \%$. This intermediate undergoing diazotization and following coupling reaction with dimethylamine led to the target compound. The overall yield was $22.3 \%$. All the intermediates and the target products dacarbazine were confirmed by ${ }^{1} \mathrm{H}$ NMR, ${ }^{13} \mathrm{C}$ NMR and ESI-MS. The current process is economic efficient characterized by applied cheap materials, mild conditions, and good overall yield.
\end{abstract}

Keywords dacarbazine; process optimization; drug synthesis; anticancer

达卡巴嗪作为细胞非特异性药物有着广谱的抗癌 活性，能够抑制肉瘤 S180、白血病 L1210 及腺癌 755 , 一 直以来是临床上用于治疗恶性黑色素瘤的首选药物 ${ }^{[1,2]}$. 达卡巴嗪在体内可以被转化为一种与嘌呤的生物合成 中间产物相似的化合物, 可能对嘌呤的生物合成具有干 扰作用. 该药进入人体后在肝微粒体的作用下，去甲基 化成为单甲基化合物, 显示出直接的细胞毒作用, 主要 作用于细胞周期的 G2 期，通过烷基化作用抑制 DNA、 RNA 及蛋白质的合成, 发挥抗肿瘤作用. 由于该药对某 些癌症的治疗有着不可或缺的地位, 近年来更有将其应
用于肿瘤的联合免疫治疗的报道 ${ }^{[3]}$. 因此，一直以来， 人们致力于该化合物合成工艺的研究，期望获得一条简 便、绿色、经济的合成路线.

据文献调研，目前达卡巴嗪的合成路线有三条 (Scheme 1). 第一条路线是由 Alexandrina 等 ${ }^{[4]}$ 报道的. 他们直接以 5-氨基-4-咪唑甲酰胺为原料, 首先将氨基 重氮化得重氮盐, 再与二甲胺偶联获得达卡巴嗪. 另外 两条合成路线其实都是对第一条路线的改进，即解决如 何合成 5-氨基-4-咪唑甲酰胺的问题, 其它步骤与路线 1 同. 作者认为, 以 4-咪唑甲酸为原料合成 5-氨基-4-咪唑

\footnotetext{
* E-mail: thrchen@jnu.edu.cn

Received October 18, 2014; revised December 19, 2014; published online January 7, 2015.

Project supported by the National Natural Science Foundation of China (No. 81172982).

国家自然科学基金(No. 81172982 )资助项目.
} 
甲酰胺的路线路线 $2^{[5]}$, 存在原料价格高、工艺过程安全 隐患大(需要催化氢化)等缺点. 而基于二氨基马来腈的 合成路线路线 $3^{[6]}$, 虽然路线简洁, 但需要用到剧毒的 氢氰酸为原料, 安全隐患更大. 另外, 二氨基马来腈的 成本也相当昂贵. 因此, 有必要探索更优化的 5-氨基-4咪唑甲酰胺合成路线.

通过逆合成分析, 5-氨基-4-咪唑甲酰胺(5)的合成以 廉价的甘氨酸和氨基腈(或尿素)为主要原料来实现 (Scheme 2). 首先是甘氨酸经甲酯化、甲酰化、脱水成 异氧、最后转酰胺化得 $\alpha$-异氧基乙酰胺(4), 该化合物在 碱性环境下与氨基腈或尿素发生串联反应和电子重排 形成咪唑环, 得关键中间体 5-氨基-4-咪唑甲酰胺(5). 每 步反应的具体实验条件均在参考相关文献 [7 12]的基 础上进一步优化而得.

\section{1 结果与讨论}

按照 Scheme 2 所示路线合成达卡巴嗪. 首先是 $\alpha$ 异氰基乙酰胺(4)的合成, 经 4 步反应完成. 第一步是甘 氨酸羧基的甲酯化, 利用过量甲醇作为底物兼溶剂, 在 二氯亚砜 $\left(\mathrm{SOCl}_{2}\right)$ 的作用下以 $93.0 \%$ 的收率获得甘氨酸 甲酯盐酸盐(1); 第二步将氨基甲酰化, 反应试剂为 $\mathrm{HCOOCH}_{3} / \mathrm{Et}_{3} \mathrm{~N}$, 室温摚拌即可反应, 收率为 $78.0 \%$; 甲 酰氨基脱水成异氰基的反应在 $\mathrm{POCl}_{3}$ 的作用下顺利进 行, 收率 65.0\%; 第四步是将 $\alpha$-异氰基乙酸酯(3)转化为 $\alpha$-异氧基乙酰胺(4), 只需要在室温条件下, 在浓度为 7 $\mathrm{mol} / \mathrm{L}$ 的 $\mathrm{NH}_{3}$ /甲醇溶液中进行反应，即可以高达 $91.0 \%$ 的收率获得酰胺化产物 4.

本文路线中，由 $\alpha$-异氧基乙酰胺(4)与适当的试剂 合成 5-氨基-4-咪唑甲酰胺 (5) 是关键步骤. 参考文献 [10 12]，作者选择氨基腈或尿素为合适的试剂在碱性 条件与化合物 4 反应获得 5 . 本实验对碱性试剂、溶剂、 温度及反应时间等因素进行探索(表 1), 发现以氨基腈 为底物, $\mathrm{NaH}$ 为碱性试剂, $\mathrm{THF}$ 为溶剂, $0{ }^{\circ} \mathrm{C}$ 投料反应, 室温下反应 $3 \mathrm{~h}$ 为最佳条件, 能够以 $68.0 \%$ 的收率获得 化合物 5(表 1, Entry 7); 同样条件下，以尿素为底物，其 收率只有 $13.0 \%$ (表 1, Entry 3). 虽然以 $t$-BuOLi 为碱性 试剂的收率略高一些(表 1, Entry 8), 但需要在较低温度 下进行, 所以我们认为, 反应条件仍以表 1 Entry 7 为佳. 起初我们选择廉价的尿素作为原料与化合物 4 合成 5, 尝试了许多实验条件, 结果都不太理想(表 1 , Entries $1 \sim 6)$. 这可以从图 1 的反应机理中得到合理的解释. 化 合物 4 首先在碱的作用下形成碳负离子中间体 $\mathbf{X}, \mathbf{X}$ 进 攻尿素的羰基形成中间体 U1. 由于尿素的羰基有两个 氨基的共轭作用，其接受亲核试剂进攻的能力被削弱， 所以由 $\mathrm{X}$ 生成 $\mathrm{U} 1$ 的过程并不十分有利. $\mathrm{U} 1$ 发生分子内 亲核加成反应，同时从体系中捕获质子转化 U2，U2 脱 水形成咪唑环的结构, 这一过程在碱性环境下, 需要比 较大的活化能才能促使反应的进行; 相比之下, 氨基腈<smiles>NC(=O)c1nc[nH]c1N</smiles><smiles>NC(=O)c1nc[nH]c1CCCCCO[N+](=O)[O-]</smiles>

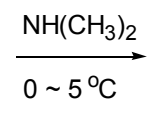<smiles>CN(C)/N=N/c1[nH]cnc1C(N)=O</smiles>
dacarbazine

Route 2:<smiles>N[N+]([O-])([O-])C(=O)c1c[nH]cn1</smiles><smiles>NC(=O)c1nc[nH]c1[N+](=O)[O-]</smiles><smiles>NC(=O)c1nc[nH]c1[N+](=O)[O-]</smiles><smiles>[PbH]</smiles><smiles>NC(=O)c1nc[nH]c1N</smiles>

Route 3: $\mathrm{HCN}+$<smiles>N#C/C(N)=C(\N)C#N</smiles><smiles>CN=CN=C(N)C#N</smiles>

$\mathrm{NaOH}$ aq

图式 1 文献报道的达卡巴嗪合成路线

Scheme 1 Synthetic processes of dacarbazine reported by literature
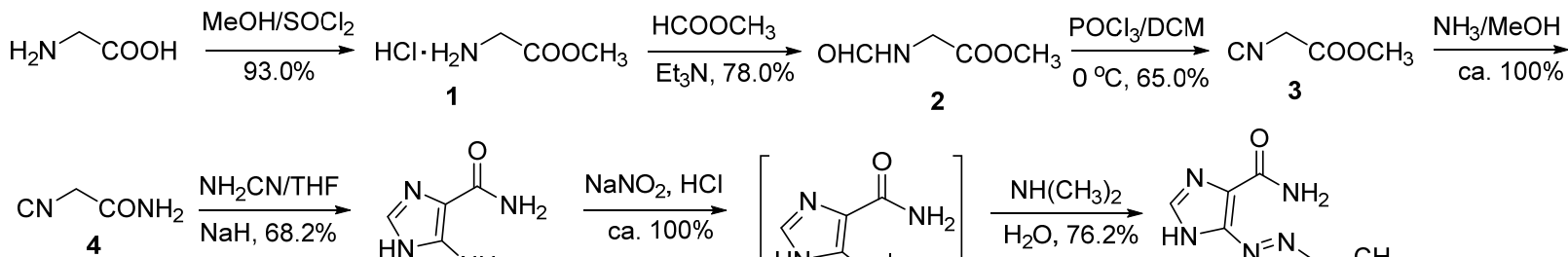<smiles>NC(=O)c1nc[nH]c1N</smiles>

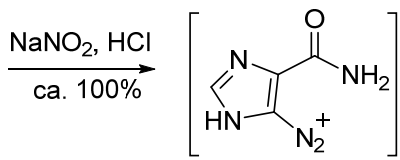

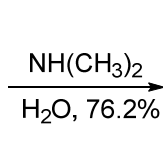<smiles>CN(C)/N=N/c1[nH]cnc1C(N)=O</smiles>

图式 2 达卡巴嗪的优化合成路线

Scheme 2 The optimized synthetic process of dacarbazine 
表 1 合成 5-氨基-4-咪唑甲酰胺的反应条件

Table 1 Synthetic conditions of 4-amino-5-imidazolecarboxamide

\begin{tabular}{|c|c|c|c|c|c|}
\hline Entry & 原料 & 碱性试剂 & 溶剂 & 温度/时间 & 收率 $/ \%$ \\
\hline 1 & 尿素 & $\mathrm{CH}_{3} \mathrm{ONa}$ & $\mathrm{CH}_{3} \mathrm{OH}$ & $0{ }^{\circ} \mathrm{C}$ r.t. $/ 4 \mathrm{~h}$ & 17.0 \\
\hline 2 & 尿素 & $\mathrm{NaH}$ & THF & $0{ }^{\circ} \mathrm{C} / 5 \mathrm{~h}$ & 0.0 \\
\hline 3 & 尿素 & $\mathrm{NaH}$ & THF & $0{ }^{\circ} \mathrm{C} \rightarrow$ r.t. $/ 5 \mathrm{~h}$ & 13.0 \\
\hline 4 & 尿素 & $\mathrm{NaH}$ & THF & $60{ }^{\circ} \mathrm{C} / 5 \mathrm{~h}$ & 0.0 \\
\hline 5 & 尿素 & $t$-BuOLi & THF & $-15{ }^{\circ} \mathrm{C} \rightarrow$ r.t. $/ 5 \mathrm{~h}$ & 30.0 \\
\hline 6 & 尿素 & $t$-BuOLi & $t-\mathrm{BuOH}$ & $-15{ }^{\circ} \mathrm{C} \rightarrow$ r.t. $/ 5 \mathrm{~h}$ & 21.0 \\
\hline 7 & 氨基腈 & $\mathrm{NaH}$ & THF & $0{ }^{\circ} \mathrm{C} \rightarrow$ r.t. $/ 3 \mathrm{~h}$ & 68.2 \\
\hline 8 & 氨基腈 & $t$-BuOLi & THF & $-15^{\circ} \mathrm{C} \rightarrow$ r.t. $/ 5 \mathrm{~h}$ & 69.3 \\
\hline
\end{tabular}

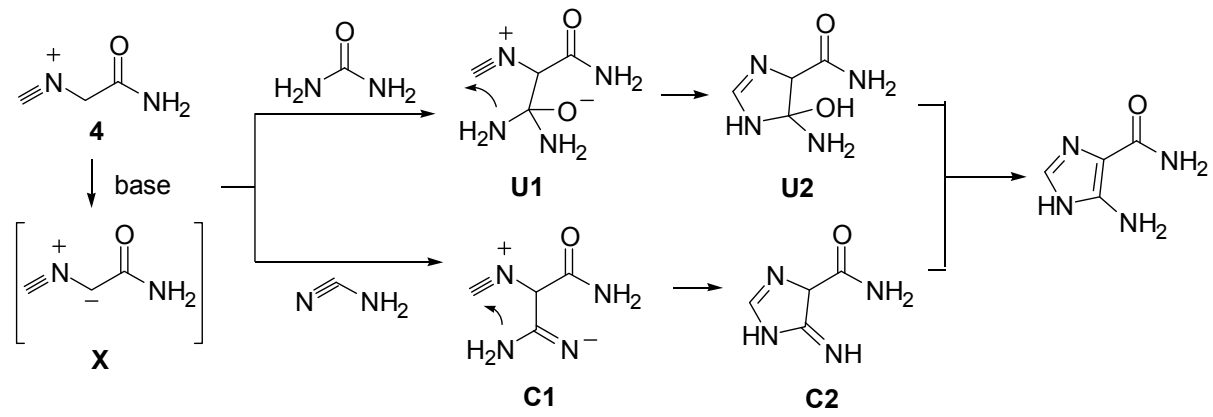

图 1 5-氨基-4-咪唑甲酰胺的形成机理

Figure 1 The formation mechanism of 4-amino-5-imidazolecarboxamide

的氰基碳为 $\mathrm{sp}$ 杂化, 其亲核加成的活性比尿素的羰基 高, 因此, 由 $\mathbf{X}$ 生成 $\mathbf{C} 1$ 比较容易进行. 更进一步地, 由 C1 发生分子内亲核加成反应形成 C2 是一个有利的过 程. 另外, C2 只需通过分子内电子重排, 将环外双键转 化为环内双键, 形成咪唑芳香体系, 这是个热力学有利 的转化, 反应活化能小. 由此可见, 氨基腈是比尿素更 为合适的、与化合物 $\mathbf{4}$ 反应形成 $\mathbf{5}$ 的反应试剂.

氨基氰是重要的农药中间体, 大量用于农药的制备 合成, 在医药工业上广泛用作生产盐酸阿糖胞苷的原 料, 价格低廉, 易于获取, 使本合成路线实现工业化生 产的可能性增加. 最后, 化合物 $\mathbf{5}$ 在 $\mathrm{NaNO}_{2} / \mathrm{HCl}$ 作用下 形成重氮盐, 然后与二甲胺耦合得到目标产物达卡巴 嗪, 收率 $76.2 \%$.

\section{2 结论}

成功地以廉价的甘氨酸为原料, 经过五步应合成了 重要前体化合物 5-氨基-4-咪唑甲酰胺, 再经过重氮化 和偶合反应成功地合成了抗肿瘤药物达卡巴嗪, 总收率 $22.3 \%$. 相对于文献报道的其它方法而言, 该路线所用 原料价廉易得、反应条件温和, 总收率较高. 同时提供 了一种高效合成取代咪坐衍生物的新方法.

\section{3 实验部分}

\section{1 仪器与试剂}

Bruker-AV300MHz 型核磁共振仪(瑞士 Bruker 公
司); DHG-9140A 型恒温干燥箱(上海浦东荣丰科学仪器 有限公司); CLJBQ-3 型恒温磁力搅拌器(郑州长城科工 贸有限公司); EYELA 型旋转蒸发仪(东京理化器械独资 工厂); LGJ-12 冷冻干燥机(巩义市英峪予华仪器有限公 司); FA2104 型电子天平(上海恒平科学仪器有限公司); TU-1810S 紫外分光光度计(北京普析通仪器有限公司); LC-UV100 液相色谱仪(上海伍丰科学仪器有限公司); DZF-150 型小型真空干燥箱(郑州长城科工贸有限公司); KQ3200E 型超声波清洗器(昆山市超声仪器有限公司); X-5 型显微熔点测定仪(北京泰克仪器有限公司). 甘氨 酸、尿素、氨基氰、二甲胺等均为 aladdin 试剂(上海晶 纯科技有限公司).

\section{2 实验方法}

\subsection{1 甘氨酸甲酯(1)的合成}

合成方法参照文献[7]. 称取甘氨酸 7.5 g (100 $\mathrm{mmol}$ )于 $500 \mathrm{~mL}$ 圆底烧瓶中, 用 $200 \mathrm{~mL}$ 重蒸过的甲醇 做溶剂, 冰浴中搅拌冷却 $15 \mathrm{~min}$. 用注射器量取 22 $\mathrm{mL}$ (约 $300 \mathrm{mmol}$ )二氯亚砜, 慢慢滴加入反应瓶中, 于室 温下反应过夜. 室温下旋转蒸除去多余的二氯亚砜和甲 醇, 残留物用尽量少的热甲醇溶解, 然后快速地加入大 量冷乙醚并将反应瓶放入冰浴中快速冷却, 此时析出大 量白色的固体，抽滤洗涤得白色固体 $11.6 \mathrm{~g}$, 收率 $93.0 \%$. m.p. $174 \sim 175{ }^{\circ} \mathrm{C}$ (文献值 ${ }^{[7]}$ m.p. $174 \sim 175{ }^{\circ} \mathrm{C}$ ); ${ }^{1} \mathrm{H}$ NMR $\left(300 \mathrm{MHz}, \mathrm{D}_{2} \mathrm{O}\right) \delta: 3.90(\mathrm{~s}, 2 \mathrm{H}), 3.78(\mathrm{~s}, 3 \mathrm{H}) ;{ }^{13} \mathrm{C}$ 
NMR (75 MHz, $\left.\mathrm{D}_{2} \mathrm{O}\right) \delta: 168.7,53.4,40.2$; MS-ESI $m / z$ : $179.2[2 \mathrm{M}+\mathrm{H}]^{+}$.

\subsection{2 $N$-甲酰甘氨酸甲酯 $(2)$ 的合成}

合成方法参照文献[8]. 称取甘氨酸甲酯 $3.56 \mathrm{~g}(0.4$ $\mathrm{mol}$ )、甲酸甲酯 $10 \mathrm{~mL}$ 、三乙胺 $5 \mathrm{~mL}$ 置于反应瓶中, 在 室温下搅拌 $3 \mathrm{~d}$ 后, 将反应瓶置于 $0{ }^{\circ} \mathrm{C}$ 下静置 $30 \mathrm{~min}$, 使固体析出, 过滤除去固体杂质; 将液体减压旋干得到 无色油状物质, TLC 检测表明纯度合格, 不需进一步分 离纯化; 将油状物置于真空干燥箱中干燥, 得无色液体 $2.95 \mathrm{~g}$, 收率为 $78.0 \%$. ${ }^{1} \mathrm{H}$ NMR $\left(300 \mathrm{MHz}, \mathrm{D}_{2} \mathrm{O}\right) \delta: 8.25$ (s, 1H), 4.15 (s, 2H), 3.83 (s, 3H); ${ }^{13} \mathrm{C}$ NMR $(75 \mathrm{MHz}$, D2O) $\delta: 171.7,164.6,52.8,39.8$; MS-ESI $m / z: 118.2[\mathrm{M}+$ $\mathrm{H}]^{+}$.

\subsection{3 $\alpha$-异氧基乙酸甲酯(3)的合成}

合成方法参照文献[9]. 将 $N$-甲酰甘氨酸甲酯 $2.75 \mathrm{~g}$ (23.5 mmol)溶解在 $100 \mathrm{~mL}$ 重蒸的无水二氯甲烷(DCM) 中, 接着加入三乙胺 $8.1 \mathrm{~mL}(58.7 \mathrm{mmol})$, 将反应混合物 置于冰浴中搅拌冷却 $15 \mathrm{~min}$. 待冷却完全后, 慢慢滴加 三氯氧磷 $2.2 \mathrm{~mL}$ (23.5 mmol)于反应体系中, 而后继续 在冰浴中搅拌反应 $1 \mathrm{~h}$. 随后将溶液升至室温, 并缓慢 加入饱和 $\mathrm{Na}_{2} \mathrm{CO}_{3}$ 溶液直至 $\mathrm{pH}$ 显示为碱性. 继续摚拌至 水相和有机相充分分层, 分液漏斗分出有机层. 水层用 $\mathrm{DCM}$ 萃取 $(30 \mathrm{~mL} \times 3)$. 合并有机层, 用饱和食盐水洗涤 3 次, 有机相用无水 $\mathrm{MgSO}_{4}$ 干燥, 过滤洗涤, 合并滤液 并旋干, 得粗品 $\alpha$-异氧基乙酸甲酯, 该粗品用 RP-HPLC 分离纯化, 真空干燥后得具极强刺激性气味的暗棕色液 体 $1.51 \mathrm{~g}$, 收率 $65.0 \% .{ }^{1} \mathrm{H}$ NMR $\left(300 \mathrm{MHz}, \mathrm{CDCl}_{3}\right) \delta$ : $4.21(\mathrm{~s}, 2 \mathrm{H}), 3.72(\mathrm{~s}, 3 \mathrm{H}) ;{ }^{13} \mathrm{C} \mathrm{NMR}\left(75 \mathrm{MHz}, \mathrm{CDCl}_{3}\right) \delta$ : 164.5, 160.5, 53.1, 43.2; MS-ESI $m / z: 98.2[\mathrm{M}-\mathrm{H}]^{-}$.

\section{$3.2 .4 \alpha$-异氧基乙酰胺(4)的合成}

合成方法参照文献[9]. 取 $\alpha$-异氧基乙酸甲酯 $1.0 \mathrm{~g}$ $(5.0 \mathrm{mmol})$ 于 $25 \mathrm{~mL}$ 圆底烧瓶中, 加入 $5.0 \mathrm{~mL}$ 甲醇, 翻 口胶塞密闭. 用注射器将溶有 $7 \mathrm{~mol} / \mathrm{L}$ 氨气的甲醇溶液 $1.5 \mathrm{~mL}(10.5 \mathrm{mmol})$ 加入反应体系中, 于室温下搅拌过 夜. 反应结束后旋蒸除去过量的氨和甲醇, 将残留物 (黄色固体)重新溶解于 $10.0 \mathrm{~mL}$ 甲醇中, 加入 $5 \%$ 的活性 炭, 于 $50{ }^{\circ} \mathrm{C}$ 下搅拌 $2 \mathrm{~h}$. 混合物通过硅藻土过滤, 将滤 液浓缩得到灰白色固体. RP-HPLC 分析表明纯度 95\%, 无需进一步分离纯化, 将产物真空干燥, 得灰白色固体 $765 \mathrm{mg}$, 收率 91.0\%. m.p. 113 114 ${ }^{\circ} \mathrm{C}$ (文献值 ${ }^{[10]} \mathrm{m} . \mathrm{p}$. $\left.113 \sim 114{ }^{\circ} \mathrm{C}\right) ;{ }^{1} \mathrm{H}$ NMR $\left(300 \mathrm{MHz}, \mathrm{D}_{2} \mathrm{O}\right) \delta: 4.71(\mathrm{~s}, 2 \mathrm{H})$, 4.37 (s, 2H); ${ }^{13} \mathrm{C}$ NMR (75 MHz, $\left.\mathrm{D}_{2} \mathrm{O}\right) \delta: 168.4,156.2$, 44.6; MS-ESI $m / z: 85.1[\mathrm{M}+\mathrm{H}]^{+}$.

\subsubsection{5-氨基-4-咪唑甲酰胺(5)的合成}

合成方法参照文献[10 12]. 取 $\mathrm{NaH}(60 \%) 280$ $\mathrm{mg}($ 约 $7.0 \mathrm{mmol})$ 于氮气保护下加入重蒸的四氢呋喃 (THF) $10 \mathrm{~mL}$, 冰浴冷却 $15 \mathrm{~min}$; 取 $420 \mathrm{mg}(5.0 \mathrm{mmol})$ $\alpha$-异氧基乙酰胺溶解于 $5 \mathrm{~mL}$ 重蒸的 THF 中, 将其滴加 到上述体系中，搅拌反应 $0.5 \mathrm{~h}$; 另取氨基氰 $210 \mathrm{mg}(5.0$ $\mathrm{mmol}$ )溶于 THF 中, 通过注射器缓慢加入到反应体系中. 加料完毕, 撤去冰浴, 使体系慢慢升至室温, 继续加班 反应 $3 \mathrm{~h}$, 过程中析出部分固体. 反应结束, 加入 $5.0 \mathrm{~mL}$ 水淬灭反应, 并用稀盐酸调节 $\mathrm{pH}$ 至 9 左右, 旋干溶剂后 用甲醇溶解残留物, 以短的固相萃取柱和 $0.25 \mu \mathrm{m}$ 滤膜 过滤样品后, 样品用 RP-HPLC 纯化[淋洗剂为 $V$ (甲 醇) $: V($ 水 $)=15: 85$ ], 合并馏分, 旋蒸除去甲醇, 残留 液冻干得灰白色固体粉末 $430 \mathrm{mg}$, 收率 $68.2 \%$. m.p. 169 171 ${ }^{\circ} \mathrm{C}$ (文献值 ${ }^{[13]}$ m.p. $168 \sim 170{ }^{\circ} \mathrm{C}$ ); ${ }^{1} \mathrm{H}$ NMR (300 MHz, DMSO- $\left.d_{6}\right) \delta: 11.45(\mathrm{br}, 1 \mathrm{H}), 7.14(\mathrm{~s}, 1 \mathrm{H}), 6.80$ $(\mathrm{s}, 2 \mathrm{H}), 5.56(\mathrm{~s}, 2 \mathrm{H}) ;{ }^{13} \mathrm{C}$ NMR $\left(75 \mathrm{MHz}\right.$, DMSO- $\left.d_{6}\right) \delta$ : 167.2, 146.1, 129.8, 109.2; MS-ESI $m / z: 127.1[\mathrm{M}+\mathrm{H}]^{+}$.

\section{2 .6 达卡巴嗪的合成}

合成方法参照文献[4]. 取 5-氨基-4-咪唑甲酰胺 378 $\mathrm{mg}(3.0 \mathrm{mmol})$ 置于 $50 \mathrm{~mL}$ 圆底烧瓶中, 加入 $1.0 \mathrm{~mL}$ 蒸馏 水冰浴搅拌; 取 $1.2 \mathrm{~mL}$ (约 $12.0 \mathrm{mmol}$ ) 浓盐酸用 $2.0 \mathrm{~mL}$ 水稀释后慢慢加入反应瓶中, 反应始终保持在冰浴下进 行; 取亚硝酸钠 $\left(\mathrm{NaNO}_{2}\right) 207 \mathrm{mg}$ (3.0 mmol)溶解在 1.0 $\mathrm{mL}$ 蒸馏水中慢慢加入到反应体系中, 用淀粉碘化钾试 纸检测确证反应过程亚硝酸钠过量. 此时反应液变成深 红色, 反应始终保持在冰浴下进行. 搅拌 $0.5 \mathrm{~h}$ 后, 往反 应体系中滴加含有 $40 \%$ 二甲胺的水溶液 $0.5 \mathrm{~mL}$ (4.5 $\mathrm{mmol}$ ), 继续低温反应 $2 \mathrm{~h}$. 反应结束, 将反应液冻干. 将冻干的残留物用水溶解后过固相色谱柱和 $0.25 \mu \mathrm{m}$ 滤 膜, 样品用 RP-HPLC 纯化[淋洗剂为 $V$ (甲醇) $: V$ (水) $=$ $15: 85$ ], 合并馏分, 低温旋蒸除去甲醇, 残留液冻干得 淡红色固体粉末 $415 \mathrm{mg}$, 收率 76.2\%. m.p. 203 205 ${ }^{\circ} \mathrm{C}$ (文献值 ${ }^{[4]}$ m.p. $203 \sim 205{ }^{\circ} \mathrm{C}$ ); ${ }^{1} \mathrm{H}$ NMR $\left(300 \mathrm{MHz}, \mathrm{D}_{2} \mathrm{O}\right.$ ) $\delta: 15.14(\mathrm{br}, 1 \mathrm{H}), 9.03(\mathrm{br}, 2 \mathrm{H}), 8.52(\mathrm{~s}, 1 \mathrm{H}), 2.51(\mathrm{~s}, 6 \mathrm{H})$; ${ }^{13} \mathrm{C}$ NMR $\left(75 \mathrm{MHz}, \mathrm{D}_{2} \mathrm{O}\right) \delta: 152.8,152.6,143.9,119.8$, 34.4; MS-ESI $m / z: 181.3[\mathrm{M}-\mathrm{H}]^{-}$.

辅助材料(Supporting Information) 化合物 1 5 及达 卡巴嗪的 ${ }^{1} \mathrm{H}$ NMR 和 ${ }^{13} \mathrm{C}$ NMR 图谱. 这些材料可以免 费从本刊网站(http://sioc-journal.cn/)上下载.

\section{References}

[1] Wexler, P. In Encyclopedia of Toxicology, 3rd ed., Ed.: Andrea, M., Manchester, 2014, pp. 1132 1134.

[2] Zheng, M.; Sun, G. J. J. Pract. Oncol. 1998, 13(6), 328 (in Chinese). 
(郑敏, 孙国君, 实用肿瘤学杂志, 1998, 13(6), 328.)

[3] Pretto, F.; Elia, G.; Castioni, N.; Neri, D. Cancer Immunol. Immunother. 2014, 63(9), 901.

[4] Alexandrina, C.; Cornea, F.; Ionescu, C. D. RO 80163, 1983 [Chem. Abstr. 1984, 101, 151849].

[5] Jain, M. L.; Tsao, Y. P.; Ho, N. L.; Cheng, J. W. J. Org. Chem. 2001, 66, 6472.

[6] Mitsuru, T.; Masami, H.; Yoshikazu, I. JP 544941, 2004 [Chem. Abstr. 2004, 354906].

[7] Chaudhary, S.; Singh, K. R. Asian J. Pharm. Clin. Res. 2012, 5(4), 196.
[8] Panella, L.; Aleixandre, A. M.; Kruidhof, G. J.; Robertus, J. J. Org. Chem. 2006, 71, 2026.

[9] Tomasz, G.; Robert, H.; Scott, H. US 13983747, 2013 [Chem. Abstr. 2012, 157, 383004].

[10] Elders, N.; Ruijter, E.; Frans, J. J. Chem. Eur. J. 2009, 15, 6096.

[11] Elders, N.; Ruijter, E.; Frans, J. J. Chem. Eur. J. 2008, 14, 4961.

[12] Murakami, T.; Otsuka, M.; Ohno, M. Tetrahedron Lett. 1982, 23(45), 4729.

[13] Saladino, R.; Crestini, C.; Ciambecchini, U.; Ciciriello, F.; Costanzo, G.; Di Mauro, E. ChemBioChem 2004, 5, 1558.

(Li, L.; Lu, Z.) 